\title{
Small-scale biophysical couplings in \\ Lake Baikal: new insights from the \\ fractal properties of temperature and phytoplankton distributions
}

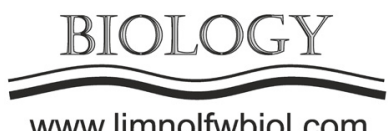

www.limnolfwbiol.com

\author{
Seuront L. ${ }^{1,2,3 *}$, Tanaka Y. ${ }^{4}$, Katano T. ${ }^{4,6}$, Sugiyama M. ${ }^{5}$, Watanabe Y. ${ }^{6}$, \\ Shimaraev M.N.7, Drucker V.V.7 \\ ${ }^{1}$ The University of Lille, CNRS, Univ. Littoral Côte d'Opale, UMR 8187, LOG, Laboratoire d'Océanologie et de Géosciences, F-59000, \\ Lille, France \\ ${ }^{2}$ Department of Marine Resources and Energy, TUMSAT, Tokyo, Japan \\ ${ }^{3}$ Department of Zoology and Entomology, Rhodes University, Grahamstown, South Africa \\ ${ }^{4}$ Department of Ocean Sciences, TUMSAT, Tokyo, Japan \\ ${ }^{5}$ Department of Natural Resources, Kyoto University, Kyoto, Japan \\ ${ }^{6}$ Faculty of Science, Tokyo Metropolitan University, Tokyo, Japan \\ ${ }^{7}$ Limnological Institute, SB RAS, Irkutsk, Russia
}

\begin{abstract}
Using vertical profiles of temperature and in vivo fluorescence sampled between Tanhoy and Listvyanka, we show that temperature and fluorescence fluctuations exhibit specific levels of internal structuration that are independent of the overall vertical structure of the water column, which suggest a previously untapped small-scale biophysical coupling.
\end{abstract}

Keywords: Couplings, plankton, passive and biologically-active scalars, turbulence, fractals

\section{Introduction}

Since the pioneering work of the late Trevor Platt (1942-2020) in the seventies, the study of biophysical couplings in lakes and oceans has been a very prolific research topic. Physical processes were often implicitly assumed to be the main factors structuring biological communities, especially in surface waters that are typically under the influence of turbulence. This essentially led to assess the nature of biological couplings through a comparison of the fluctuations of passive scalars (e.g. temperature, salinity, and a priori phytoplankton cells), which have widely been described in Fourier space using power spectral analysis as $E(f) \propto f^{\beta}$ where $f$ is the frequency $\left(\mathrm{s}^{-1}\right)$ or the wavelength $\left(\mathrm{m}^{-1}\right)$ and $\beta=5 / 3$ for passive scalars, and $\beta \neq 5 / 3$ for biologically active scalars. From the mid-nineties, fractals and multifractals have been proposed as a new tool to assess the complexity of passive and biologically active scalars; see Seuront (2010) for a review. These methods, together with the increase in both spatial and temporal resolutions of physical and biological sensors, increasingly led to identify distinct structures in the fluctuations of physical and biological scalars. In this context, to illustrate the applicability of the fractal approach in lakes, we used a set of temperature and in vivo fluorescence vertical profiles taken across Lake Baikal in August 2003 between Tanhoy and
Listvyanka to compare the nature of temperature and phytoplankton fluctuations under different scenario of vertical distributions (Fig.).

\section{Materials and Methods}

We investigated the properties of both temperature and fluorescence fluctuations using a method derived from geostatistics and regionalized variables based on the behavior of the semivariance $\gamma(h)$ defined as:

$$
\gamma(h)=\frac{1}{2 N(h)} \sum_{i=1}^{N(h)}[Z(i)-Z(i+h)]^{2}
$$

where $Z(i+h)$ is the value of the dependent variable $z(i)$ at a depth separated from depth $i$ by the distance, or lag, $h$, and $N(h)$ is the number of pairs of data points separated by the lag $h$. Because semivariance analysis is based on the assumption of at least reduced stationarity, our analyses were based on the first difference of both temperature and fluorescence, i.e. $Z(i)=[\mathrm{d} x / \mathrm{d} z]$, where $x$ is either temperature or fluorescence and $z$ the depth. If the semivariance is a power-law of the scale $h$, i.e. $\gamma(h) \propto h^{m}$, where $m$ is the slope of the log-log plot of $\gamma(h)$ versus $h$, the fractal dimension $D(D=(4-m)$ $/ 2, D \in[1,2])$ can be used as an index of the degree of spatial structure of $z(i)$. Specifically, the limits 


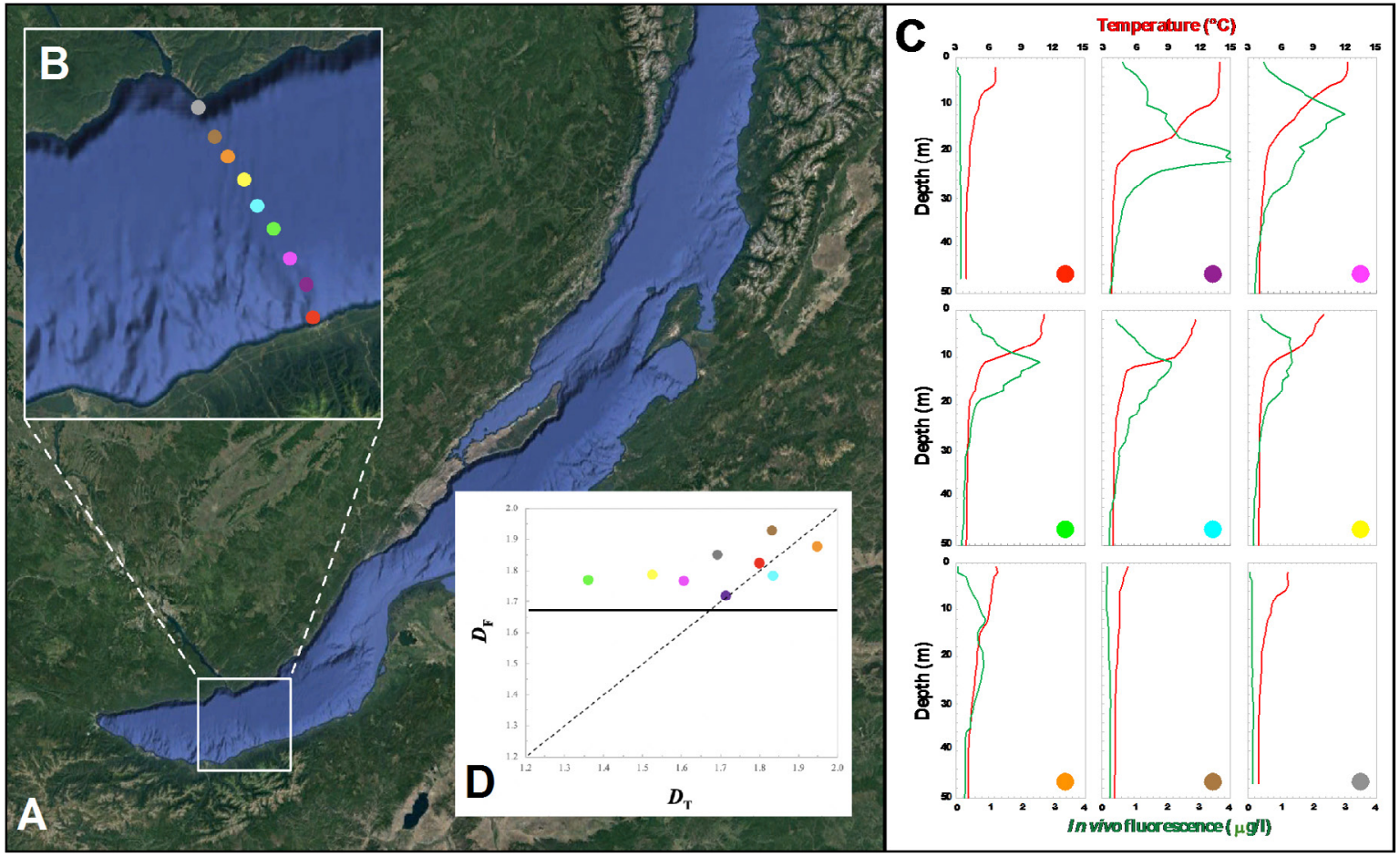

Fig. Study area on the Lake Baikal (A), location (B) and vertical structure of temperature and in vivo fluorescence (C) profiles analyzed in the present work, and (D) the fractal dimension $D_{\mathrm{F}}$ of fluorescence fluctuations shown as a function of the fractal dimension $D_{\mathrm{T}}$ of temperature fluctuations. Each of the 9 color codes used in (B), (C) and (D) consistently refer to a specific vertical profile.

$D=1$ and $D=2$ characterized vertical distributions where the differences between nearby measurements are respectively higher than and no different from the differences between distant samples.

\section{Results and Discussion}

All the vertical profiles investigated showed fractal properties, irrespective of the actual vertical structure of the water column (Fig.). The fractal dimensions of temperature fluctuations $\left(D_{\mathrm{T}}\right)$ ranged between 1.36 and 1.95 and were significantly $(p$ $<0.01$ ) smaller than the fractal dimensions of fluorescence $\left(D_{\mathrm{F}}\right)$ distributions, which ranged from 1.72 and 1.93. Noticeably, $D_{\mathrm{T}}$ and $D_{\mathrm{F}}$ were consistently significantly larger than the theoretical value $D=1.67$ expected for a passive scalar under the influence of three-dimensional fully developed turbulence. Finally,
$D_{\mathrm{F}}$ were significantly positively correlated with $D_{\mathrm{F}}$, i.e. $D_{\mathrm{F}}=0.19 D_{\mathrm{T}}+1.48\left(r^{2}=0.29, p<0.05\right)$.

These preliminary results show that vertical profiles of both physical and biological parameters present specific levels of internal structure that are independent of the overall vertical structure of the water column. More specifically, they indicate (i) a stronger spatial structure in phytoplankton than in temperature, (ii) a lack of control of both temperature and phytoplankton by turbulent velocity fluctuations, and (iii) the existence of a potential causal link between temperature and phytoplankton fluctuations, hence a form of small-scale biophysical coupling.

\section{References}

Seuront L. 2010. Fractals and multifractals in ecology and aquatic science. Boca Raton: CRC Press. 